\title{
Trapped interacting two-component bosons in one dimension
}

\author{
Shi-Jian $\mathrm{Gu}^{1}$, You-Quan $\mathbf{L i}^{1,2}$ and Zu-Jian Ying ${ }^{1}$ \\ ${ }^{1}$ Zhejiang Institute of Modern Physics, Zhejiang University, Hangzhou 310027, P.R. \\ China \\ ${ }^{2}$ Institute for Physics, Augsburg University, D-86135 Augsburg, Germany
}

\begin{abstract}
In this paper we solve one dimensional trapped $\mathrm{SU}(2)$ bosons with repulsive $\delta$-function interaction by means of Bethe-ansatz method. The features of ground state and low-lying excited states are studied by numerical and analytic methods. We show that the ground state is an isospin "ferromagnetic" state which differs from spin- $1 / 2$ fermions system. There exist three quasi-particles in the excitation spectra, and both holon-antiholon and holon-isospinon excitations are gapless for large systems. The thermodynamics equilibrium of the system at finite temperature is studied by thermodynamic Bethe ansatz. The thermodynamic quantities, such as specific heat etc. are obtained for the case of strong coupling limit.
\end{abstract}

PACS numbers: 03.65.Ge, 02.20.-a, 71.10.-w 


\section{Introduction}

In the past few years, Bose Einstein condensation (BEC) has witnessed a sequence of exhilarating experimental achievements, and much attention has been paid to the study of Bose systems. The possibility of BEC in one-dimension has been discussed for the non-interacting Bose gas [1, 2]. The role of dimensionality has been examined for the ideal Bose gas [3]. It is known the interaction between bosons plays an essential role in one dimension due to the strong constraint in phase space [4]. The Luttinger liquid properties [5] of trapped, interacting quasi-one-dimensional Bose gas was discussed [6]. It was shown under suitable experimental conditions that the system can be described as a Luttinger liquid and the correlation function of the bosons decays algebraically which prevents Bose-Einstein condensation. Recently, a two component Bose gas was produced in magnetically trapped ${ }^{87} R b$ by rotating the two hyperfine states into each other with the help of slightly detuned Rabi oscillation field [7, 8]. It was noticed [9] that the properties of the Bose system can be different from the traditional scalar Bose system once it acquires internal degree of freedom. The two-component Bose system on a circle (periodic boundary conditions) has been studied by means of Bethe-ansatz method [10]. It was shown that the ground state is an isospin-ferromagnetic state which differs from the one-dimensional spin-1/2 Fermi system whose ground state is $\mathrm{SU}(2)$ singlet.

In this paper, we solve trapped two-component bosons with $\delta$-function interaction in one dimension by Bethe-ansatz method. On the basis of Bethe-ansatz equation, we discuss the ground state, low-lying excited states and the thermodynamics of the system at finite temperature, where some thermal quantities at low temperature are obtained explicitly. Our paper is organized as follows: In the next section we introduce the model and derive a set of non-linear equations for charge rapidity and isospin rapidity. In Sec. 3, we explicitly show that the ground state is an isospin "ferromagnetic" state and manifest how the quantum numbers in Bethe-ansatz equation should be taken for the ground state. In Sec. 4 , We study the low-lying excited states extensively by analyzing the possible variations in the sequence of quantum numbers. Numerical results of energy"momentum" spectra for each excitation are given. In Sec. 5 we discuss the general thermodynamics of the system by means of thermodynamic Bethe ansatz [13]. In Sec.

6 we derive the free energy and specific heat in the case of strong coupling limit. In the last section a brief summary is given.

\section{The model and its Bethe-ansatz solution}

The Hamiltonian for a two-component bosons trapped in a potential well of infinite depth reads

$$
H=-\sum_{i=1}^{N} \frac{\partial^{2}}{\partial x_{i}^{2}}+\sum_{i=1}^{N} V\left(x_{i}\right)+2 c \sum_{i>j=1}^{N} \delta\left(x_{i}-x_{j}\right)
$$


where

$$
V\left(x_{i}\right)= \begin{cases}0 & |x| \leq L / 2 \\ \infty & |x|>L / 2\end{cases}
$$

The Hamiltonian is not invariant under translation due to the presence of trapping potential, and the total momentum of the system is not conserved. However, the system is still invariant under the action of the permutation group $S_{N}$ which makes it possible to employ the coordinate Bethe-ansatz approach.

In the domain with $x_{i} \neq x_{j}(i \neq j)$ and inside the potential well, the Hamiltonian reduces to the one for free bosons and it's eigenfunctions are therefore just the superpositions of plane waves. When two particles collide with each other at the same point, a scattering process happens. The Bethe-ansatz embodies that this process is purely elastic and the occurrence during the process is merely that the particles exchange their momenta. So for a given momentum $k=\left(k_{1}, k_{2}, \ldots, k_{N}\right)$, the scattering momenta include all permutations of the components of $k$. Moreover, since the total momentum is not conserved in the present model, we will have more nondiffractive scattering momenta, e.g., for $N=2$, we have $\left(k_{1}, k_{2}\right) \rightarrow\left(-k_{1}, k_{2}\right),\left(k_{1},-k_{2}\right),\left(-k_{1},-k_{2}\right)$, $\left(k_{2}, k_{1}\right),\left(-k_{2}, k_{1}\right),\left(k_{2},-k_{1}\right)$, or $\left(-k_{2},-k_{1}\right)$. All the eight states correspond to the same energy $k_{1}^{2}+k_{2}^{2}$. Hence, besides all possible permutations of the components of $k$, the scattering must include all possibilities of sign changes in the components of $k$.

Now we consider the case of $N$ bosons. Considering the fact that the total momentum is not conserved, we adopt the following Bethe-ansatz form [14]

$$
\psi_{a}(x)=\sum_{P \in \mathcal{W}_{\mathcal{B}}} A_{a}(P, Q) e^{i(P k \mid Q x)} x \in \mathcal{C}(Q)
$$

where $a=\left(a_{1}, a_{2}, \ldots, a_{N}\right), a_{j}$ denotes the isospin label of the $j$ th particle; $P k$ stands for the image of a given $k:=\left(k_{1}, k_{2}, \cdots, k_{N}\right)$ by a mapping $P \in \mathcal{W}_{B}$ and the coefficients $A(P, Q)$ are functionals on $\mathcal{W}_{B} \otimes \mathcal{W}_{A}$. Here, $\mathcal{W}_{B}$ and $\mathcal{W}_{A}$ stand for the Weyl group of $B_{N}$ and $A_{N-1}$ Lie algebras respectively. The later is isomorphic to the permutation group $S_{N}$ and the former consists of $S_{N}$ and all possible sign changes. We emphasize that the sum runs over the Weyl group of Lie algebra $B_{N}$ but the wave function is defined on various Weyl chambers $\mathcal{C}(Q)$ corresponding to the Weyl group of $A_{N-1}$ Lie algebra. This is different from the situation of periodic boundary conditions.

For a Bose system, the wave function is supposed to be symmetric under any permutation of both coordinates and isospin indices, i.e.

$$
\left(\sigma^{j} \psi\right)_{a}(x)=\psi_{a}(x)
$$

Here $\left(\sigma^{j} \psi\right)_{a}$ is well defined by $\psi_{\sigma^{j} a}\left(\sigma^{j} x\right)$, therefore both sides of (4) can be written out by using (3). Furthermore, using the evident identity $\left(P k \mid \sigma^{i} Q x\right)=\left(\sigma^{i} P k \mid Q x\right)$ and the rearrangement theorem of group theory, we obtain the following consequence from (4)

$$
A_{a}\left(P, \sigma^{i} Q\right)=A_{\sigma^{j} a}\left(\sigma^{i} P, Q\right) .
$$


The $\delta$-function term in the Hamiltonian (1) contributes a boundary conditions at hyperplane $\mathbf{P}_{\alpha}$ ( $\alpha$ is a root of Lie algebra $A_{N-1}$ ), namely a discontinuity of the derivatives of the wave function along the normal of a Weyl hyperplane:

$$
\lim _{\epsilon \rightarrow 0^{+}}\left[\alpha \cdot \nabla \psi_{a}\left(x_{(\alpha)}+\epsilon \alpha\right)-\alpha \cdot \nabla \psi_{a}\left(x_{(\alpha)}-\epsilon \alpha\right)\right]=2 c \psi_{a}\left(x_{(\alpha)}\right)
$$

where $x_{(\alpha)} \in \mathbf{P}_{\alpha}$ and $\nabla:=\sum_{i=1}^{N} e_{i}\left(\partial / \partial x_{i}\right)$. Substituting (3) into (6), we find that

$$
\begin{aligned}
& i\left[(P k)_{j}-(P k)_{j+1}\right]\left[A_{a}\left(P, \sigma_{i} Q\right)-A_{a}\left(\sigma_{i} P, \sigma_{i} Q\right)-A_{a}(P, Q)+A_{a}\left(\sigma_{i} P, Q\right)\right] \\
& =c\left[A_{a}(P, Q)+A_{a}\left(\sigma_{i} P, Q\right)+A_{a}(P, Q)+A_{a}\left(\sigma_{i} P, Q\right)\right]
\end{aligned}
$$

By making use of the relation (5) and the continuity relation of wave function across $(Q x)_{i}=(Q x)_{i+1}$, we can obtain the following relations

$$
\begin{aligned}
& A_{a}\left(\sigma_{i} P, Q\right)=\check{S}_{a, a^{\prime}}^{i}(P k) A_{a^{\prime}}(P, Q) \\
& \check{S}_{a, a^{\prime}}^{i}(P k)=-\frac{c \delta_{a, a^{\prime}}+i\left[(P k)_{i}-(P k)_{i+1}\right] \mathcal{P}_{a, a^{\prime}}}{c-i\left[(P k)_{i}-(P k)_{i+1}\right]}
\end{aligned}
$$

where $\mathcal{P}_{a, a^{\prime}}$ stands for the matrix elements of the spinor representation of the permutation group. The relation (4) provides for the coefficients $A$ a relation between different Weyl chambers. Eq.(8) provides a connection between those coefficients which are related via any element of Weyl group $\mathcal{W}_{B}$ in the same Weyl chamber.

The basic elements of the Weyl group $\mathcal{W}_{B}$ obey $\sigma^{i} \sigma^{i}=1$ and $\sigma^{i} \sigma^{i+1} \sigma^{i}=$ $\sigma^{i+1} \sigma^{i} \sigma^{i+1}$ as identities. These identities imply that $A_{a}\left(\sigma^{i} \sigma^{i} P, Q\right)=A_{a}(P, Q)$ and $A_{a}\left(\sigma^{i} \sigma^{i+1} \sigma^{i} P, Q\right)=A_{a}\left(\sigma^{i+1} \sigma^{i} \sigma^{i+1} P, Q\right)$. Using (8) repeatedly, one can obtain the following relations:

$\check{S}^{i}\left(\sigma^{i} P k\right) \check{S}^{i}(\sigma k)=I$

$\check{S}^{i}\left(\sigma^{i+1} \sigma^{i} P k\right) \check{S}^{i+1}\left(\sigma^{i} P k\right) \check{S}^{i}(P k)=\check{S}^{i+1}\left(\sigma^{i} \sigma^{i+1} P k\right) \check{S}^{i}\left(\sigma^{i+1} P k\right) \check{S}^{i+1}(P k)$

where we have adopted the conventions $\check{S}=\operatorname{mat}\left(S_{a b}\right), \check{S}^{i}=\check{S} \otimes I, \check{S}^{i+1}=I \otimes \check{S}(I$ is a $2 \times 2$ unit matrix). These relations are consistency conditions for the $\mathrm{S}$-matrix. The second relation is called the Yang-Baxter equation. The concrete S-matrix in (9) fulfills these relations.

Due to the infinite depth of the potential well, the wave function must vanish at the ends of the well,

$$
\psi_{a}\left((Q x)_{1}=-L / 2\right)=0, \quad \psi_{a}\left((Q x)_{N}=L / 2\right)=0
$$

These boundary conditions give rise to

$$
\begin{aligned}
& A_{a}\left(\tau^{1} P, Q\right)=-e^{-i(P k)_{1} L} A_{a}(P, Q) \\
& A_{a}(\gamma P, Q)=-e^{i(P k)_{N} L} A_{a}(P, Q)
\end{aligned}
$$

where $\gamma=\sigma^{N-1} \ldots \sigma^{1} \tau^{1} \sigma^{1} \ldots \sigma^{N-1}$. The relation (12) together with eq. (8) determine the amplitudes $A^{\prime}$ 's up to an overall factor since $\sigma^{1}, \ldots, \sigma^{N-1}$ and $\tau^{1}$ generate the whole $\mathcal{W}_{B}$. There are two further consistency conditions. One comes from the identity 
$\left(\tau^{1} \sigma^{1} \tau^{1} \sigma^{1}=\sigma^{1} \tau^{1} \sigma^{1} \tau^{1}\right)$ of the Weyl group $\mathcal{W}_{B}$ leading to a reflection related Yang-Baxter equation which is fulfilled automatically due to the fact that the reflection matrix is just the unit matrix multiplied by a scalar function. The other one comes from eq.(13) which leads to an eigenvalue equation for the products of the S-matrices. This eigenequation can be diagonalized by means of standard quantum inverse scattering method[15]. One finally obtains the following Bethe-ansatz equations

$$
\begin{gathered}
e^{i 2 k_{j} L}=\prod_{l \neq j}^{N} \frac{k_{j}-k_{l}+i c}{k_{j}-k_{l}-i c} \frac{k_{j}+k_{l}+i c}{k_{j}+k_{l}-i c} \prod_{\mu=1}^{M} \frac{k_{j}-\lambda_{\mu}-i c / 2}{k_{j}-\lambda_{\mu}+i c / 2} \frac{k_{j}+\lambda_{\mu}-i c / 2}{k_{j}+\lambda_{\mu}+i c / 2} \\
1=\prod_{l=1}^{N} \frac{\lambda_{\gamma}-k_{l}-i c / 2}{\lambda_{\gamma}-k_{l}+i c / 2} \frac{\lambda_{\gamma}+k_{l}-i c / 2}{\lambda_{\gamma}+k_{l}+i c / 2} \prod_{\mu \neq \gamma}^{M} \frac{\lambda_{\gamma}-\lambda_{\mu}+i c}{\lambda_{\gamma}-\lambda_{\mu}-i c} \frac{\lambda_{\gamma}+\lambda_{\mu}+i c}{\lambda_{\gamma}+\lambda_{\mu}-i c}
\end{gathered}
$$

where $M$ denotes the total number of down isospins and $\lambda$ denote isospin rapidities which arise from the diagonalization conditions of quantum inverse scattering method. Taking the logarithm of eqs. (14) gives rise to the secular equations:

$\pi I_{j}=$

$k_{j} L+\sum_{l \neq j}^{N}\left[\tan ^{-1}\left(\frac{k_{j}-k_{l}}{c}\right)+\tan ^{-1}\left(\frac{k_{j}+k_{l}}{c}\right)\right]-\sum_{\mu=1}^{M}\left[\tan ^{-1}\left(\frac{k_{j}-\lambda_{\mu}}{c / 2}\right)+\tan ^{-1}\left(\frac{k_{j}+\lambda_{\mu}}{c / 2}\right)\right]$

$\pi J_{\gamma}=$

$\sum_{l=1}^{N}\left[\tan ^{-1}\left(\frac{\lambda_{\gamma}-k_{l}}{c / 2}\right)+\tan ^{-1}\left(\frac{\lambda_{\gamma}+k_{l}}{c / 2}\right)\right]-\sum_{\mu \neq \gamma}^{M}\left[\tan ^{-1}\left(\frac{\lambda_{\gamma}-\lambda_{\mu}}{c}\right)+\tan ^{-1}\left(\frac{\lambda_{\gamma}+\lambda_{\mu}}{c}\right)\right]$

where $I_{j}$ is the quantum number for the charge rapidity $k_{j}$ and $J_{\gamma}$ for the isospin rapidity. Concerning the property of the logarithm function, $I_{j}$ and $J_{\gamma}$ take integer values regardless of either the total number of particles or that of isospin down. Once the roots are solved from eqs. (15) for a given set of quantum numbers $\left\{I_{j}, J_{\gamma}\right\}$, the energy can be calculated by $E=\sum_{j=1}^{N} k_{j}^{2}$.

\section{The ground state}

For any set of quantum numbers $\left\{I_{j}, J_{\gamma}\right\}$ with the solution $\left\{k_{j}, \lambda_{\gamma}\right\}$, the replacement of either $I_{j} \rightarrow-I_{j}, k_{j} \rightarrow-k_{j}$ or $J_{\gamma} \rightarrow-J_{\gamma}, \lambda_{\gamma} \rightarrow-\lambda_{\gamma}$ keeps the secular equation (15) invariant. So it makes no change to the energy, then we only need to consider the case of positive integer [16] for $I s$ and $J s$. In the weak coupling limit $c \rightarrow 0$, $\tan ^{-1}(x / c) \rightarrow \pi \operatorname{sgn}(x) / 2$, eqs. (15) become

$$
\begin{aligned}
& \pi I_{j}=k_{j} L+\frac{\pi}{2} \sum_{l \neq j}^{N}\left[\operatorname{sgn}\left(k_{j}-k_{l}\right)+\operatorname{sgn}\left(k_{j}+k_{l}\right)\right]-\frac{\pi}{2} \sum_{\mu=1}^{M}\left[\operatorname{sgn}\left(k_{j}-\lambda_{\mu}\right)+\operatorname{sgn}\left(k_{j}+\lambda_{\mu}\right)\right] \\
& 2 J_{\gamma}=\sum_{l=1}^{N}\left[\operatorname{sgn}\left(\lambda_{\gamma}-k_{l}\right)+\operatorname{sgn}\left(\lambda_{\gamma}+k_{l}\right)\right]-\sum_{\mu \neq \gamma}^{M}\left[\operatorname{sgn}\left(\lambda_{\gamma}-\lambda_{\mu}\right)+\operatorname{sgn}\left(\lambda_{\gamma}+\lambda_{\mu}\right)\right]
\end{aligned}
$$


We may choose both the subscript of the $J_{j}$ and $J_{\gamma}$ in an increasing order so that the second equation of eqs. (16) becomes

$$
\sum_{l=1}^{N} \operatorname{sgn}\left(\lambda_{\gamma}-k_{l}\right)=2 J_{\gamma}+2 \gamma-N-2
$$

which also gives rise to

$$
\sum_{l=1}^{N}\left[\operatorname{sgn}\left(\lambda_{\gamma+1}-k_{l}\right)-\operatorname{sgn}\left(\lambda_{\gamma}-k_{l}\right)\right]=2\left(J_{\gamma+1}-J_{\gamma}\right)+2
$$

Thus, for $J_{\gamma+1}-J_{\gamma}=m$, there must exist exactly $m+1$ momenta of $k_{l}$ satisfying $\lambda_{\gamma}<k_{l}<\lambda_{\gamma+1}$.

Similarly, from the first equation of eqs. (16) we have

$$
2\left(I_{j+1}-I_{j}\right)-\frac{2 L}{\pi}\left(k_{j+1}-k_{j}\right)-2=-\sum_{\mu=1}^{M}\left[\operatorname{sgn}\left(k_{j+1}-\lambda_{\mu}\right)-\operatorname{sgn}\left(k_{j}-\lambda_{\mu}\right)\right](
$$

For $I_{j+1}-I_{j}=n$, there will be $k_{j+1}-k_{j}=(n+m-1) \pi / L$ if there are $m \lambda_{\gamma}$ satisfying $k_{j}<\lambda_{\gamma}<k_{j+1}$. Therefore, an isospin rapidity of value $\lambda_{\mu}$ always repels the quasimomentum away from that value. The ground state for $c \rightarrow 0$ does not allow any possible existence of $\lambda_{\mu}$ between $k_{1}$ and $k_{N}$. Thus from eq. (17) and eq. (18) we can conclude that the total number of isospin down should be zero. The quantum numbers $\left\{I_{j}^{0}\right\}$ of the ground state are given by the successive positive numbers $I_{1}^{0}=1, I_{j+1}^{0}-I_{j}^{0}=1$. In the thermodynamic limit, as the difference between two adjacent $k$ 's is small, the contribution of any existing $\lambda$ to the density of $k$-rapidity at points $k=\lambda$ will brings about a rift (see fig.1 right). Therefore, an existing $\lambda_{\mu}$ will suppress the density of state in $k$-space at the point $k=\lambda_{\mu}$. The more isospin rapidities there are, the higher the energy will be. In contrast to the spin-1/2 Fermi system whose ground state is $\mathrm{SU}(2)$ singlet, the ground state of trapped 2-component bosons is an isospin "ferromagnetic" state which is described by the quantum number,

$$
\left\{I_{j}\right\}:=\{1, \cdots, N\},\left\{J_{\gamma}\right\}=\text { empty }
$$

Since the existence of open boundary conditions makes the backwards scattering $k \rightarrow-k$ possible, there exists an additional term $\tan ^{-1}(2 k / c)$ which contributes a negative value to $\rho(k)$, especially $\rho(0)$ is obviously suppressed. This phenomenon is different from that for the system with periodic conditions. We interpret it being due to the confinement by the infinite-depth well, for example, the ground state for a single particle is not $k=0$ but $k=\pi / L$ in open boundary conditions. The density of states of the ground state for various coupling is plotted in fig.1 the left.

By introducing

$$
\begin{aligned}
& L \rho\left(\frac{k_{j}+k_{j+1}}{2}\right)=\frac{1}{k_{j+1}-k_{j}} \\
& L \sigma\left(\frac{\lambda_{\gamma}+\lambda_{\gamma+1}}{2}\right)=\frac{1}{\lambda_{\gamma+1}-\lambda_{\gamma}}
\end{aligned}
$$


the density corresponding to the quantum numbers (20) satisfies the integral equation

$$
\rho_{0}(k)=\frac{1}{\pi}-\frac{1}{L} K_{1 / 2}(k)+\int_{0}^{Q_{0}} K_{1}\left(k \mid k^{\prime}\right) \rho_{0}\left(k^{\prime}\right) d k^{\prime}
$$

where

$$
K_{n}(x)=\frac{1}{\pi} \frac{n c}{n^{2} c^{2}+x^{2}}
$$

and

$$
K_{n}(x \mid y)=K_{n}(x-y)+K_{n}(x+y)
$$

$\rho_{0}, Q_{0}$ are respectively the density and integration limit for the ground state. The $K_{1 / 2}(k)$ term in (22) comes from the fact that $\tan ^{-1}\left(\frac{k_{j}-k_{l}}{c}\right) \rightarrow 0$ but $\tan ^{-1}\left(\frac{k_{j}+k_{l}}{c}\right) \rightarrow$ $\tan ^{-1}\left(\frac{k_{j}}{c / 2}\right)$ when taking account of the term of $l=j$ in the process of thermodynamic limit. The particle number per length is determined by

$$
D=\frac{N}{L}=\int_{0}^{Q_{0}} \rho_{0}(k) d k
$$

eqs. (22) and eq. (23) determine $Q_{0}$ and $\rho_{0}(k)$. Then the energy can be calculated by

$$
\frac{E_{0}}{L}=\int_{0}^{Q_{0}} k^{2} \rho(k) d k
$$

which is explicitly $\frac{1}{3} \pi^{2} D^{3}\left(1-\frac{4}{c} D\right)$ in the strong coupling limit $c \gg 1$. In the general case one needs to solve the equations numerically. We show the ground state energy for various densities $D=1.0,0.75,0.5$ in fig.2.

\section{Low-lying excited states}

The excited states are obtained by the variation of the configuration $\left\{I_{j}, J_{\gamma}\right\}$ from that of the ground state. The simplest case is to remove one of $I s$ from the configuration of the ground state and add a new $I_{n}$ outside the original sequence. We call this holonantiholon excitation which is described by

$$
\left\{I_{j}\right\}=\left\{1, \cdots, n-1, n+1, \ldots, N, I_{n}\right\}
$$

with $\left|I_{n}\right|>N$ and $M=0$. To investigate the excited states, we shall consider systems of finite size first, then take thermodynamics limits. Although the total momentum $\sum_{j} k_{j}$ is no more a constant in open boundary conditions, $\sum_{j}\left|k_{j}\right|$ is still a constant for a given energy eigenstate. We plotted the numerical results for energy- "momentum" spectrum in fig. 3 , and 1 for $N=40, L=40$ where the $x$-axis represents the quantitive change $\sum_{j}\left(\left|k_{j}\right|-\left|k_{j}^{0}\right|\right)$. We notice that the overall structure of the spectrum does not change substantially between weak $(c=1)$ and strong $(c=10)$ coupling regimes. For a finite-size system, the gap of holon-antiholon excitation opens, and it's dependence on the inter-particle interaction is shown by the upper line of fig.5.

In the thermodynamic limit, it is plausible to calculate the excitation energy by making $\rho(k)=\rho_{0}(k)+\rho_{1}(k) / L$ where $\rho_{0}(k)$ is the density of the ground state. By 
creating a hole inside the quasi Fermi sea $\bar{k} \in\left[0, Q_{0}\right]$ and an additional $k_{p}>Q_{0}$ outside it, we have

$$
\rho_{1}(k)+\delta(k-\bar{k})=\int_{0}^{Q_{0}} d k^{\prime} \rho_{1}\left(k^{\prime}\right) K_{1}\left(k \mid k^{\prime}\right)+K_{1}\left(k \mid k_{p}\right)
$$

The excitation energy consists of two terms $\triangle E=\int k^{2} \rho(k) d k-\int k^{2} \rho_{0}(k) d k=$ $\varepsilon_{h}(\bar{k})+\varepsilon_{a}\left(k_{p}\right)$. The holon energy $\varepsilon_{h}$ and antiholon energy $\varepsilon_{a}\left(k_{p}\right)=-\varepsilon_{h}\left(k_{p}\right)$ are given by

$$
\begin{aligned}
& \varepsilon_{h}(\bar{k})=-\bar{k}^{2}+\int_{0}^{Q_{0}} k^{2} \rho_{1}^{h}(k, \bar{k}) d k, \\
& \rho_{1}^{h}(k, \bar{k})=-K_{1}(k \mid \bar{k})+\int_{0}^{Q_{0}} K_{1}\left(k \mid k^{\prime}\right) \rho_{1}^{h}\left(k^{\prime} \mid \bar{k}\right)
\end{aligned}
$$

Another interesting excitation is to flip one isospin down, i.e., $M=1$. As discussed above, a single $\lambda$ can suppress the density of state at $k=\lambda$. We plot the density of the lowest energy state for various couplings in fig.1 the right with $N=100, L=100$ and $J_{1}=30$. The holon-isospinon excitation is characterized by the Young tableau $[N-1,1]$, accordingly, $I$ 's take $N$ distinct integers and $I_{1}<J_{1}<I_{N}$, namely

$$
\begin{gathered}
I_{1}=-N / 2+\delta_{1, j_{1}} \quad\left(1 \leq j_{1} \leq N+1\right) \\
I_{j}=I_{j-1}+1+\delta_{j, j_{1}} \quad(j=2, \ldots, N)
\end{gathered}
$$

where $\delta_{\alpha, \beta}$ is the usual Kronecker symbol. The excitation spectra are plotted in fig. $\oplus$, for $L=20, N=20, c=10$ and $c=1$ respectively. The lowest energy state of one isospin down is to make the absolute value of $J_{1}$ as large as possible in order to avoid the enhancement of energy caused by the suppression of the $k$-distribution. In the thermodynamic limit, it can be shown that this mode is gapless. For a finite particle system, however, it still has a gap of order $1 / L$ which decreases more quickly than that for periodic conditions for particle number ranging from small to large. Figure 5 shows it's dependence of the gap on the interaction. We presented in fig. 6 the behavior of the finite-size spin gap as a function of $1 / L$ at $c=1.0,10.0,100.0$.

In the thermodynamic limit, we should take into account of the hole in the $k$-sector and the $\rho_{1}(k)$ should be solved from

$$
\rho_{1}(k)+\delta(k-\bar{k})=\int K_{1}\left(k \mid k^{\prime}\right) \rho_{1}\left(k^{\prime}\right) d k^{\prime}-K_{1 / 2}(k \mid \lambda)
$$

One can find that the energy of holon-isospinon excitation consists of two terms $\triangle E=\varepsilon_{h}(\bar{k})+\varepsilon_{\text {iso }}(\lambda)$. The $\varepsilon_{h}$ is determined by eqs. (26) and the $\varepsilon_{\text {iso }}$ by $\varepsilon_{\text {iso }}(\lambda)=$ $\int k^{2} \rho_{1}^{i s o}(k, \lambda) d k$ with

$$
\rho_{1}^{i s o}(k, \lambda)=-K_{1 / 2}(k \mid \lambda)+\int_{0}^{Q_{0}} K_{1}\left(k \mid k^{\prime}\right) \rho_{1}^{i s o}\left(k^{\prime}, \lambda\right) d k^{\prime}
$$




\section{Thermodynamics at finite temperature}

For the ground state (i.e. at zero temperature), the charge rapidity $k$ 's are real roots

of the Bethe-ansatz equations (14). For the excited state, however, the isospin rapidity can be complex roots 111] which always form a "bound state" with several other $\lambda$ 's. This arises from the consistency of both sides of the Bethe-ansatz equations [12]. The $n$-string of $\lambda$ rapidity is defined as

$$
\Lambda_{a}^{n j}=\lambda_{a}^{n}+(n+1-2 j) i c / 2+O(\exp (-\delta N))
$$

where $j=1,2 \cdots n$. The number of total down isospin is determined by

$$
M=\sum_{n=1}^{\infty} n M_{n}
$$

where $M_{n}$ denotes the number of n-strings. The eqs. (15) become

$$
\begin{aligned}
\pi I_{j}= & k_{j} L+\sum_{l \neq j}^{N}\left[\tan ^{-1}\left(\frac{k_{j}-k_{l}}{c}\right)+\tan ^{-1}\left(\frac{k_{j}+k_{l}}{c}\right)\right] \\
& -\sum_{a n}\left[\tan ^{-1}\left(\frac{k_{j}-\lambda_{a}^{n}}{n c / 2}\right)+\tan ^{-1}\left(\frac{k_{j}+\lambda_{a}^{n}}{n c / 2}\right)\right] \\
\pi J_{a}^{n}= & \tan ^{-1}\left(\frac{\lambda_{a}^{n}}{n c / 2}\right)+\sum_{l=1}^{N}\left[\tan ^{-1}\left(\frac{\lambda_{a}^{n}-k_{l}}{n c / 2}\right)+\tan ^{-1}\left(\frac{\lambda_{a}^{n}+k_{l}}{n c / 2}\right)\right] \\
& -\sum_{m b t} A_{m n t}\left[\tan ^{-1}\left(\frac{\lambda_{a}^{n}-\lambda_{b}^{m}}{t c / 2}\right)+\tan ^{-1}\left(\frac{\lambda_{a}^{n}+\lambda_{b}^{m}}{t c / 2}\right)\right]
\end{aligned}
$$

where

$$
A_{n m t}= \begin{cases}1, & \text { for } t=m+n,|m-n|(\neq 0) \\ 2, & \text { for } t=n+m-2, \cdots,|n-m|+2 \\ 0, & \text { otherwise }\end{cases}
$$

and the quantum numbers $\left\{I_{j}, J_{a}^{n}\right\}$ label the state beyond the ground state. The densities corresponding to charge rapidity and isospin rapidity on the real axis for which the "omitted $k, \lambda$ values" must be taken into account. By introducing the density of holes for charge $\rho^{h}(k)$ and isospin n-string $\sigma_{n}^{h}(\lambda)$, we have

$$
\begin{aligned}
\rho+\rho^{h}= & \frac{1}{\pi}-\frac{1}{L} K_{1 / 2}(k)+\int K_{1}\left(k \mid k^{\prime}\right) \rho\left(k^{\prime}\right) d k^{\prime} \\
& -\sum_{n} \int K_{n / 2}(k \mid \lambda) \sigma_{n}(\lambda) d \lambda \\
\sigma_{n}+\sigma_{n}^{h}= & \frac{1}{L} K_{n / 2}(\lambda)+\int K_{n / 2}(\lambda \mid k) \rho(k) d k \\
& -\sum_{m t} A_{m n t} \int K_{t / 2}\left(\lambda \mid \lambda^{\prime}\right) \sigma_{m}\left(\lambda^{\prime}\right) d \lambda^{\prime}
\end{aligned}
$$

where the integration limits are $[0, \infty]$. In terms of the distribution functions of charge and isospin rapidities, the energy per length has the form $E_{k} / L=\int k^{2} \rho(k) d k$, the total 
number of down isospins is $M / L=\sum_{n} n \int \sigma_{n}(\lambda) d \lambda$ and the particle density of the system is $D=N / L=\int \rho(k) d k$.

If we consider the energy arising from the external field $\Omega$ which is the Rabi field in two-component BEC experiments, the internal energy of the system is

$$
E / L=\int\left(k^{2}-\Omega\right) \rho(k) d k+\sum_{n} 2 n \Omega \int \sigma_{n} d \lambda
$$

For a given $\rho(k), \rho^{h}(k), \sigma_{n}(\lambda)$ and $\sigma_{n}^{h}(\lambda)$, the entropy is of the form [13]

$$
\begin{aligned}
S / L= & \int\left[\left(\rho+\rho^{h}\right) \ln \left(\rho+\rho^{h}\right)-\rho \ln \rho-\rho^{h} \ln \rho^{h}\right] d k \\
& +\sum_{n} \int\left[\left(\sigma_{n}+\sigma_{n}^{h}\right) \ln \left(\sigma_{n}+\sigma_{n}^{h}\right)-\sigma_{n} \ln \sigma_{n}-\sigma_{n}^{h} \ln \sigma_{n}^{h}\right] d \lambda
\end{aligned}
$$

where the Boltzmann constant is set to unity.

At finite temperature, we should minimize the free energy $F=E-T S-\mu N$ where $\mu$ is the chemical potential and $S$ is the entropy of the system. Making use of the relations derived from eqs. (32)

$$
\begin{aligned}
& \delta \rho^{h}=-\delta \rho+\int K_{1}\left(k \mid k^{\prime}\right) \delta \rho d k^{\prime}-\sum_{n} \int K_{n / 2}(k \mid \lambda) \delta \sigma_{n} d \lambda \\
& \delta \sigma_{n}^{h}=-\delta \sigma_{n}+\int K_{n / 2}(\lambda \mid k) \delta \rho d k-\sum_{m t} A_{m n t} \int K_{t / 2}\left(\lambda \mid \lambda^{\prime}\right) \delta \sigma_{m}\left(\lambda^{\prime}\right) d \lambda^{\prime}
\end{aligned}
$$

we obtain the following conditions from the minimum condition $\delta F=0$,

$$
\begin{aligned}
\epsilon(k) & =k^{2}-\Omega-\mu-T \int K_{1}\left(k \mid k^{\prime}\right) \ln \left(1+e^{-\epsilon\left(k^{\prime}\right) / T}\right) d k^{\prime} \\
& -T \sum_{n} \int K_{n / 2}(k \mid \lambda) \ln \left[1+e^{-\zeta_{n}(\lambda) / T}\right] d \lambda \\
\zeta_{n}(\lambda) & =2 n \Omega+T \int K_{n / 2}(k \mid \lambda) \ln \left[1+e^{-\epsilon(k) / T}\right] d k \\
& +T \sum_{m t} A_{m n t} \int K_{t / 2}\left(\lambda \mid \lambda^{\prime}\right) \ln \left[1+e^{-\zeta_{m}\left(\lambda^{\prime}\right) / T}\right] d \lambda^{\prime}
\end{aligned}
$$

where we have written

$$
\begin{aligned}
& \frac{\rho^{h}(k)}{\rho(k)}=e^{\epsilon(k) / T} \\
& \frac{\sigma_{n}^{h}(\lambda)}{\sigma_{n}(\lambda)}=e^{\zeta_{n}(\lambda) / T} .
\end{aligned}
$$

When $T \rightarrow 0$, eqs. (36) become

$$
\mathcal{E}_{0}(k)=k^{2}-\Omega-\mu+\int K_{1}\left(k \mid k^{\prime}\right) \mathcal{E}_{0}\left(k^{\prime}\right) d k^{\prime}
$$

where the "ferromagnetic" ground state is under consideration. Clearly, the integral equation gives the solution for the dressed energy [17] from which the ground-state energy can be given in terms of $\mathcal{E}_{0}$

$$
E_{0} / L=\frac{1}{\pi} \int_{0}^{Q_{0}} \mathcal{E}_{0}(k) d k
$$


The solution of eq. (38) defines the energy bands. And the Fermi surface is determined by

$$
\mathcal{E}_{0}\left(k_{F}\right)=0
$$

since the ground-state configuration corresponds to the case that all states of $\mathcal{E}_{0}(k)<0$ are fully filled. The bare energy $\mathcal{E}_{0}^{(0)}$ is the zero order term of eq. (38)

$$
\mathcal{E}_{0}^{(0)}=k^{2}-\Omega-\mu
$$

which is valid in the strong coupling limit

Equations (36) can be solved by iteration. The coupled equations (32) of density distribution of charge and isospin are then a Fredholm type

$$
\begin{aligned}
\rho\left(1+e^{\epsilon / T}\right)= & \frac{1}{\pi}-\frac{1}{L} K_{1 / 2}(k)+\int K_{1}\left(k \mid k^{\prime}\right) \rho\left(k^{\prime}\right) d k^{\prime} \\
& -\sum_{n} \int K_{n / 2}(k \mid \lambda) \sigma_{n}(\lambda) d \lambda \\
\sigma_{n}\left(1+e^{\zeta_{n} / T}\right)= & \frac{1}{L} K_{n / 2}(\lambda)+\int K_{n / 2}(\lambda \mid k) \rho(k) d k \\
& -\sum_{m t} A_{m n t} \int K_{t / 2}\left(\lambda \mid \lambda^{\prime}\right) \sigma_{m}\left(\lambda^{\prime}\right) d \lambda^{\prime}
\end{aligned}
$$

Finally, we obtain the Helmholtz free energy $F=E-T S$

$$
\begin{aligned}
F= & \mu N-T \int \ln \left[1+e^{-\epsilon / T}\right]\left[\frac{L}{\pi}-K_{1 / 2}(k)\right] d k \\
& -T \sum_{n} \int \ln \left[1+e^{-\zeta_{n} / T}\right] K_{n / 2}(\lambda) d \lambda
\end{aligned}
$$

and the pressure

$$
P=-\frac{\partial F}{\partial L}=\frac{T}{\pi} \int \ln \left[1+e^{-\epsilon / T}\right] d k,
$$

which is formally the same as Yang and Yang's expression but the equations determining $\epsilon$ and $\zeta$ are different. Consequently, the partition function is given by $Z=e^{-F / T}$. The thermodynamic functions, such as partition function $Z$, free energy $F$, are of importance for a thermal system. Given either of them, one is able to calculate all thermodynamic properties for the system in principle. However, the eqs. (36) are so complicated that it is impossible to obtain the explicit form of $\epsilon$ and $\zeta$ for general case. Moreover, we can also obtain some plausible results for some special cases in the next section.

\section{Special cases}

In general, the free energy of our model should be calculated using formula (43), where $\epsilon(k)$ and $\zeta_{n}(\lambda)$ are determined from eqs. (36). Eqs. (36) can be solved by numerical method via iteration. In this section, we will only discuss the case of strong coupling at low temperature. 
When $c \rightarrow \infty, K_{n}\left(k \mid k^{\prime}\right)=0$ and $K_{n}(k)=0$, then we have

$$
\epsilon=k^{2}-\Omega-\mu
$$

and the free energy becomes

$$
F / L=\mu D-\frac{T}{\pi} \int \ln \left[1+e^{-\epsilon / T}\right] d k
$$

which can be solved by integration by part [18],

$$
F / L=\mu D-\frac{2}{\pi}\left(\frac{1}{3} \mu^{3 / 2}+\frac{T^{2} \pi^{2}}{24 \mu^{1 / 2}}\right)
$$

where the external field is set to zero.

We can not derive the specific heat directly from the afore-obtained free energy because the chemical potential is a function of temperature. From eqs. (32), the density of charge rapidity has the form

$$
\rho=\frac{1}{\pi} \frac{1}{1+e^{\left(k^{2}-\Omega-\mu\right) / T}}
$$

Clearly, at zero temperature, the quasi-Fermi surface is just the square root of the chemical potential, so we have $\mu_{0}=\pi^{2} D^{2}$ which denotes the chemical potential at zero temperature. At low temperature, however, it is determined by

$$
D=\frac{1}{\pi} \int_{0}^{\infty} \frac{1}{1+e^{\left(k^{2}-\mu\right) / T}} d k
$$

we have

$$
\mu=\pi^{2} D^{2}\left[1+\frac{\pi^{2} T^{2}}{24 \mu^{2}}\right]^{-2}
$$

The second term in the bracket is small at low temperature, so we can replace $\mu$ by $\mu_{0}$. The equation becomes

$$
\mu=\mu_{0}\left[1-\frac{\pi^{2} T^{2}}{24 \mu_{0}^{2}}\right]^{-2}
$$

then the free energy becomes

$$
F / L=\mu_{0} D\left[1+\frac{\pi^{2} T^{2}}{12 \mu_{0}^{2}}\right]-\frac{2}{3 \pi} \mu_{0}^{3 / 2}\left[1+\frac{\pi^{2} T^{2}}{4 \mu_{0}^{2}}\right]
$$

Since by thermodynamics $S=-\partial F / \partial T$ and $C_{v}=T \partial S / \partial T$, we find the specific heat at low temperature is Fermi-liquid like

$$
S=C_{v}=\frac{T}{6 D}
$$

It is the same as the result of the one-component case, since for the strong coupling limit the isospin and charge are decoupled, the contribution of isospinon to the free energy vanishes. In fig.6, the finite-size energy gap of holon-isospinon in strong coupling limit tends to zero. 


\section{Conclusions}

In this paper, we have solved a system of one dimensional trapped $\mathrm{SU}(2)$ bosons with $\delta$ function interaction by means of the Bethe-ansatz method. On the basis of Bethe-ansatz equations we first discussed the ground state of the system and found that the ground state is an isospin "ferromagnetic" state which differs greatly from the spin- $1 / 2$ fermion systems. We studied the low excitation states by both numerical and analytic methods. It was shown that there are three elementary excitation modes, and the holon-antiholon and holon-isospinon excitations are gapless for large systems. For finite system, we not only plotted some excitation spectra but also plotted the dependence of finite-size energy gap on the inter-particle interaction. The thermodynamics of the system were studied by using the thermodynamic Bethe ansatz [13. For strong coupling we found that the system exhibits the Fermi-liquid behavior, i.e. the specific heat is a linear function of $T$ at low temperature.

\section{Acknowledgment}

This work is supported by Trans-Century Training Program Foundation for the Talents and EYF98 of China Ministry of Education. YQL is also supported by AvH-Stiftung. SJG thanks D.Yang and YQL thanks K.Marzlin for helpful discussions. We would like to thank the referees for their helpful suggestions.

\section{References}

[1] Bagnato V and Kleppner D 1991 Phys. Rev. A 447439

[2] Ketterle W and Druten N J 1996 Phys. Rev. A 54656

[3] Druten N J and Ketterle W 1997 Phys. Rev. Lett. 79549

[4] Lieb E H and Liniger W 1963 Phys. Rev. 1301605 Lieb E H 1963 Phys. Rev. 130, 1616

[5] Haldane F D M 1981 Phys. Rev. Lett. 471840

[6] Monien H, Linn M and Elstner N 1998 Phys. Rev. A 58 R3395

[7] Williams J E and Holland M J 1999 Nature 401568

[8] Williams J E, Holland M J, Wieman C E and Cornell E A 1999 Phys. Rev. Lett. 833358

[9] Ho T L 1998 Phys. Rev. Lett. 81742

[10] Li Y Q, Gu S J, Ying Z J and Eckern U submitted to PRL

[11] Takahashi M 1971 Prog. Theor. Phys. 461388

[12] Woynarovich F 1982 J. Phys. C: Solid State Phys. 1585 Woynarovich F 1982 J. Phys. C: Solid State Phys. 1597

[13] Yang C N and Yang C P 1969 J. Math. Phys. 101115

[14] Li Y Q 1995 Phys. Rev. A 52, 65 Li Y Q and Gruber C 1998 Phys. Rev. Lett 801034

[15] Faddev L D, in Recent Advances in Field Theory and Statistical Mechanics, ed. Zuber J and Stora (Elsevier, Amsterdam 1984) p. 569.

[16] Gaudin M 1971 Phys. Rev. A 4386 (1971)

[17] Frahm H and Korepin V E 1990 Phys. Rev. B42 10553

[18] Gu S J, Li Y Q and Lin H Q 2000 J. Phys. A: Math. Gen. 336779 

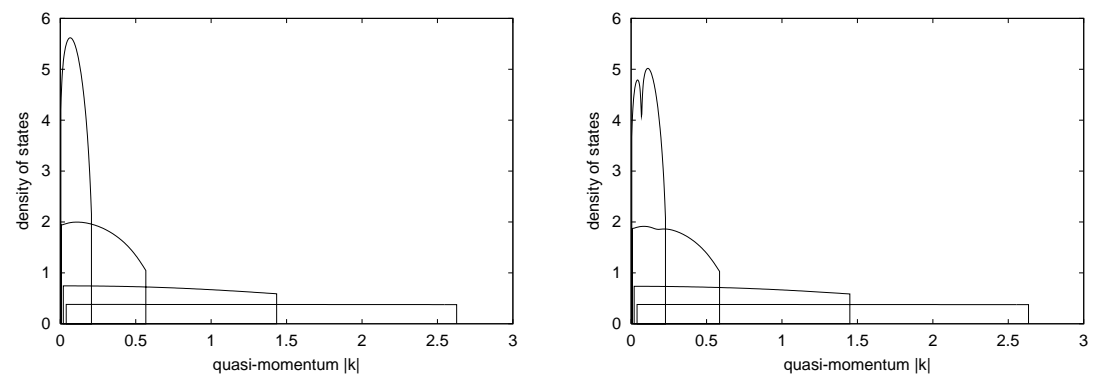

Figure 1. The density of state in $|k|$-space for the ground state (left). The distribution changes from a histogram to a narrow peak gradually for the coupling from strong to weak. The density of state in $|k|$-space in the presence of one isospin rapidity at $J_{1}=30$ where a rift evident appeared (right). The figure is plotted for $N=L=100$ and $c=10,1,0.1,0.01$.

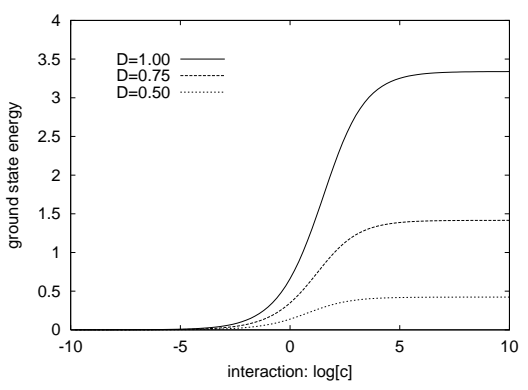

Figure 2. The ground state energy versus the coupling for different densities $D=1.0,0.75,0.5$.
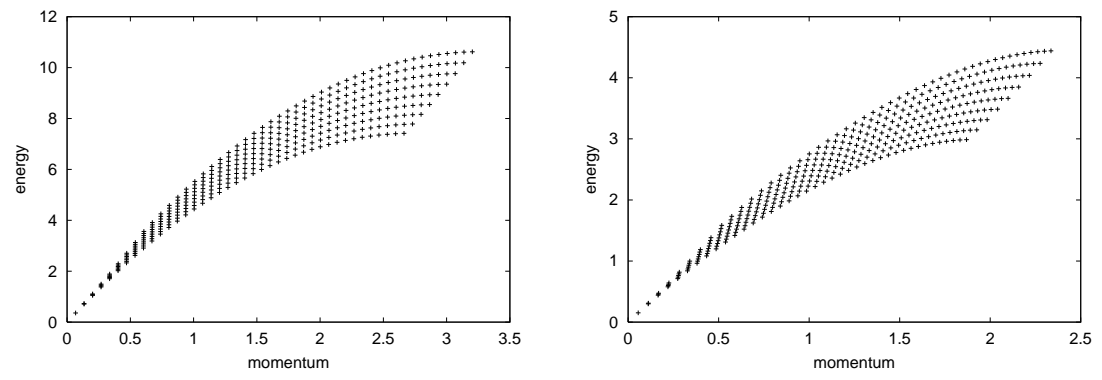

Figure 3. The holon-antiholon excitation spectrum calculated for $N=L=40$ and $c=10$ (left) and $c=1$ (right). 

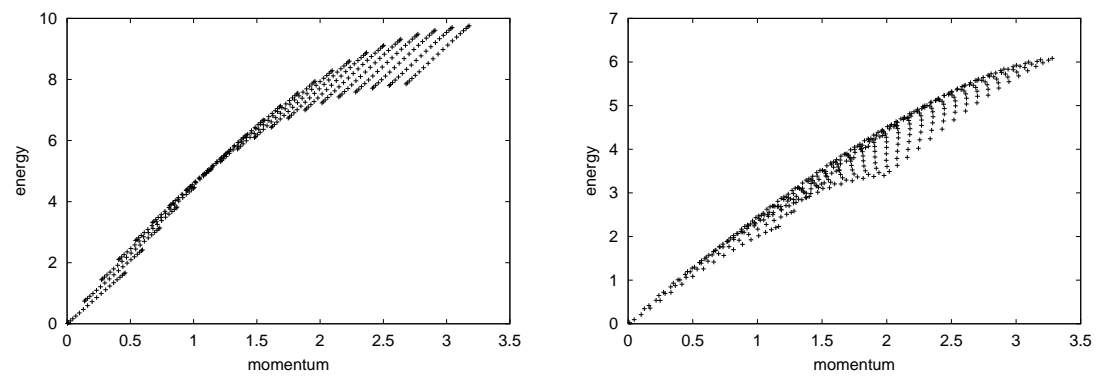

Figure 4. The holon-isospinon excitation spectrum calculated for $N=L=20, c=10$ (left) and $c=1$ (right).

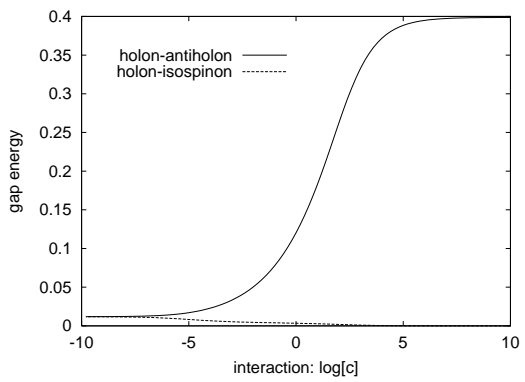

Figure 5. The finite-size energy gap versus interaction for $N=L=50$, obviously, $c=0$ corresponds to $0.02=1 / \mathrm{L}$

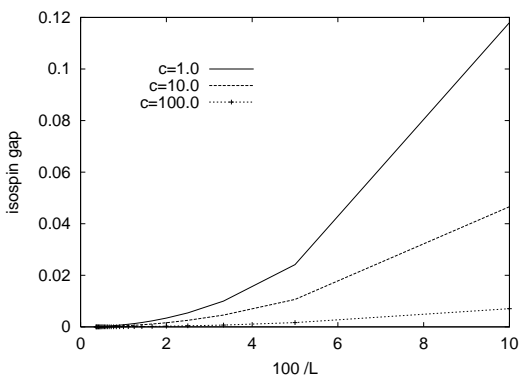

Figure 6. Finite-size effects: Isospin gap as a function of $100 / \mathrm{L}$ for $D=N / L=1$, plotted for various inter-particle interaction 